\title{
Does prolonged anti-inflammatory therapy reduce number of unnecessary repeat saturation prostate biopsy?
}

\author{
Giuseppe Candiano, Pietro Pepe, Francesco Pietropaolo, Francesco Aragona \\ Urology Unit - Cannizzaro Hospital, Catania, Italy.
}

\begin{abstract}
「summary
Introduction. The effect of a prolonged oral anti-inflammatory therapy on PSA values in patients with persistent abnormal PSA values after negative prostate biopsy (PBx) was evaluated.

Material and methods. From September 2011 to September 2012, 70 patients (median age 62 years), with persistent abnormal PSA values after negative extended PBX, were given an herbal extract with anti-inflammatory activity for 3 months (Lenidase ${ }^{\circledR} ; 1$ tablet daily constituted of baicalina, bromelina and escina). All patients were submitted to prostate biopsy for: abnormal DRE; PSA > $10 \mathrm{ng} / \mathrm{mL}$, PSA values between 4.1-10 or 2.6-4 $\mathrm{ng} / \mathrm{mL}$ with free/total $P S A<25 \%$ and $<20 \%$, respectively. Three months after the end of anti-inflammatory therapy all patients were revaluated; indication for repeat saturation biopsy (SPBX) and detection rate for PCa were compared with those previously recorded in our Department using the same inclusions criteria for biopsy.

Results. Oral administration of Lenidase ${ }^{\circledR}$ was well tolerated and no side effects were observed; PSA values decreased in $54(77.8 \%)$ out 70 patients with a median PSA reduction of $20.5 \%$ (from 8.8 to $7 \mathrm{ng} / \mathrm{mL}$ ) and remained unchanged in 16 patients (22.2\%); the repeat SPBx rate resulted significantly lower $(22.8 \%$ vs $35.5 \%$; $p<0.05)$ showing a superimposable detection rate for PCa (3 cases) in comparison with our previous data (18.7\% vs $22 \%)$.

Conclusions. In our preliminary data a prolonged oral anti-inflammatory therapy reduced PSA levels in patients with negative $\mathrm{PB} x$ and persistent suspicious for PCa decreasing the indication to perform repeat SPBx (about $30 \%$ of the cases).
\end{abstract}

KEY WORDS: Prostate cancer; Repeat saturation biopsy; Prostatitis; PSA; Anti-inflammatory therapy.

\section{INTRODUCTION}

The low specificity of PSA test in diagnosing prostate cancer (PCa), especially for serum PSA values $<10$ $\mathrm{ng} / \mathrm{mL}$ is due to spontaneous fluctuation (1), ejaculation, $\mathrm{BPH}$ and acute or subclinical chronic prostatitis (CP). PSA levels have been correlated with the extent and degree of inflammation $(2,3)$ and a short trial of antibiotics for a likely subclinical CP has some theoretical advantages in decreasing PSA levels (34.6-56\% of the cases) $(4,5)$ thus minimizing the number of biopsies for falsely elevated PSA $(20-30 \%$ of the cases) $(6,7)$.

The difference between pre and post-treatment PSA levels appears significant only in benign conditions (BPH and prostatitis cases) while in histologically proven cancer the difference seems unremarkable (8). At the same time, the administration of nonsteroidal antiinflammatory drugs (NSAIDs) or aspirin, blocking the cyclooxygenase (COX) activity (a strong mediator of inflammation), could have a potential role in decreasing PSA values. However, the relationship between oral NSAIDs consumption, PSA levels and PCa risk is unknown (9).

In this prospective study we evaluated, in patients with negative extended prostate biopsy and persistent abnormal PSA values, the effect of a prolonged oral antiinflammatory therapy on PSA values in order to reduce the number of unnecessary repeat biopsies. 


\section{Material AND METHOdS}

From September 2011 to September 2012, 70 patients (median age 62 years; range: 49-72 years), with previous negative extended prostate biopsy and persistent abnormal PSA values, were given an herbal extract with antiinflammatory activity (Lenidase ${ }^{\circledR}, 1$ tablet daily) for 3 months. All patients, 2 months before assuming the herbal extract, underwent prostate biopsy for: abnormal digital rectal examination (DRE); PSA > $10 \mathrm{ng} / \mathrm{mL}$, PSA values between $4.1-10$ or $2.6-4 \mathrm{ng} / \mathrm{mL}$ with free/total PSA $<25 \%$ and $<20 \%$, respectively according to our early diagnosis protocol (10). The biopsy was performed by transperineal approach using a $18 \mathrm{G}$ tru-cut needle guided by a $5-6.5 \mathrm{MHz}$ biplanar transrectal probe (GE Logiq 500 Pro). In case of primary biopsy or repeat saturation biopsy (SPBx) a median of 18 (range 16-21) and 28 cores (range 26-35) were taken, respectively. The biopsy protocol included a median of 9-12 cores in the posterior zone of each lobe (Apex, Med and Base) plus 2-4 cores on the transition zone in case of SPBx (11). The procedure was performed under sedation and antibiotic prophylaxis. All patients had negative DRE and no-one was symptomatic for acute prostatitis. Patients with previous HGPIN or ASAP were not included; moreover, all patients signed an informed consent form.

Clinical parameters of the patients enrolled in the protocol are listed in Table 1; 37 (52.8\%), 18 (25.7\%) and 15 (22.5\%) men previously underwent one, two and three negative biopsies, respectively. Fifty-eight (64.5\%) patients assumed alpha-blockers; none were currently treated with NSAIDs, aspirin, thiazide diuretic, statins, and 5-alfa-reductase inhibitors. At the end of the 3months course of therapy and after additional 3 months of wash-out, all patients were revaluated with DRE, total PSA, PSA F/T and routine blood test for liver and kidney function. International Prostate Symptoms Score (IPSS) and $\mathrm{Q}_{\max }$ before and after anti-inflammatory therapy were recorded. The patients with persistent suspicious for PCa underwent SPBX.

The repeat SPBx rate after anti-inflammatory therapy and detection rate for $\mathrm{PCa}$ were compared with those previ- ously recorded in our Department using the same inclusions criteria for biopsy. Finally, a p value $<0.05$ was considered statistically significant.

\section{RESULTS}

Oral administration of Lenidase ${ }^{\circledR}$ was well tolerated and no side effects were observed. Among 70 patients enrolled, PSA values decreased after anti-inflammatory therapy in $54(77.8 \%)$ with a median PSA reduction of $20.5 \%$ (from 8.8 to $7 \mathrm{ng} / \mathrm{mL}$ ) and remained unchanged in the remaining 16 patients (22.2\%). Clinical parameters and serum exams before and after Lenidase ${ }^{\circledR}$ administration are listed in Table 1. All 16 patients whose PSA did not decrease after therapy underwent repeat SPBx (6 had a PSA included between 4-10 ng/mL and 10 greater than $10 \mathrm{ng} / \mathrm{mL}$ ) and in 3 of them a cancer was found (all these men had one previous negative biopsy and a PSA > $10 \mathrm{ng} / \mathrm{mL}$ ). The incidence of repeat biopsy in the patients submitted to anti-inflammatory therapy resulted significantly lower in comparison with our previous data ( $22.8 \%$ vs $35.5 \%$ ) ( $\mathrm{p}<0.05)$, respectively; on the contrary, the detection rate for $\mathrm{PCa}$ was superimposable $(18.7 \%$ vs $22 \%)$ at repeat SPBx. IPSS and $\mathrm{Q}_{\max }$ values were superimposable before and after anti-inflammatory therapy ( $\mathrm{p}>0.05)$ (Table 1).

\section{Discussion}

Repeat prostate biopsy constitutes about 30\% of all the procedures with an estimated detection rate for $\mathrm{PCa}$ equal, in our experience, to $20 \%$ and $6 \%$ at second and third SPBx (10), respectively; today, the main goal of any early diagnosis protocol should be to reduce the number of unnecessary SPBx due to false positive PSA levels. The intra-individual (physiological) variation of PSA in men with benign prostate biopsy is equal to $9.5 \%$ (12); moreover, many common medications have an effect on serum PSA levels: NSAIDs, thiazide diuretics and statins reduce PSA levels from $6 \%$ to $26 \%$ and the combination of statins with thiazide diuretics could decrease PSA lev-

Table 1.

Clinical parameters at baseline and 6 months after anti-inflammatory therapy.

\begin{tabular}{|c|c|c|c|c|}
\hline 70 patients & Baseline & After therapy & $\%$ of reduction & p value \\
\hline Median PSA (ng/mL) & 8.8 (range: 4.6-28) & 7 (range: $2.9-23$ ) & 20.5 & $<0.05$ \\
\hline Decreased PSA levels & - & 54 & 77.2 & $<0.05$ \\
\hline$\overline{\text { PSA }>10}$ & 14 & 10 & 28.5 & $<0.05$ \\
\hline PSA 4-10 & 54 & 40 & 26.0 & $<0.05$ \\
\hline PSA $<4$ & 0 & 20 & 28.5 & $<0.05$ \\
\hline IPSS (range) & $13(4-26)$ & $12(5-24)$ & - & $>0.05$ \\
\hline Low (0-7) & 36 & 38 & & $>0.05$ \\
\hline Intermediate (8-19) & 25 & 23 & & $>0.05$ \\
\hline Severe (20-37) & 9 & 9 & & $>0.05$ \\
\hline$\overline{\mathbf{Q}_{\max }}$ & $11 \mathrm{ml} / \mathrm{sec}$ & $12 \mathrm{ml} / \mathrm{sec}$ & - & $>0.05$ \\
\hline Urine test & Negative & Negative & - & - \\
\hline Blood test & Normal & Normal & - & - \\
\hline
\end{tabular}


els of 36\% after 5 years of combined therapy (13-15). Prostatitis is responsible for falsely elevated PSA levels, but it remains unclear in which patients we can avoid repeat biopsy without underestimating the risk of cancer (16). Singer et al. (17) in 1319 men aged > 40 years observed that PSA levels were 24\% lower among acetaminophen users compared with non drug takers. Several systematic reviews have examined the relationship between NSAID/aspirin consumption and the risk of developing PCa. Mahmud et al. (18) in a meta-analysis found that aspirin use provided a 10\% risk reduction for $\mathrm{PCa}$ and a 30\% risk reduction for advanced disease. Harris et al. (19) and Jacobs et al. (20) reported that daily NSAID use (including aspirin) was associated with a $39 \%$ and 20\% risk reduction for PCa, respectively. Engelhardt and Rjedl (21) reported in 20 patients, assuming a daily dose $(60 \mathrm{mg})$ of an isoflavone extract for 1 year, a PSA reduction of 33\% combined with a significant increase in hepatic transaminases. All these studies agree that the reduction in PSA levels due to anti-inflammatory therapy may reduce the number of men referred for prostate biopsy decreasing the number of men diagnosed with PCa. However, a clear correlation between NSAIDs, serum PSA and risk of missing a PCa diagnosis is still unknown (16).

In our study, we used as anti-inflammatory agent a mixture of herbal extract (Lenidase ${ }^{\circledR}$ ) which has a demonstrated antiedematous and anti-inflammatory activity. Each tablet contains: baicalina (190 mg), a flavonoid from Scutellaria baicalensis that inhibits 5-lipoxygenase (5-LOX) and COX activities and leukotriene synthesis (22-24); bromelina (50 mg), extract from Ananas comosus, that is an enzymatic anti-inflammatory agent and escina (30 mg), extract from Aesculus hyppocastanum, that has an antiedemigenous effect and enhances corticosteroid receptors activity.

A median PSA reduction of about 20\% was observed in $54 / 70(77.2 \%)$ patients assuming Lenidase ${ }^{\circledR}$. In details, $15(28.5 \%)$ men reached a PSA value $<4 \mathrm{ng} / \mathrm{mL}$ and the repeat SPBx rate resulted significantly lower in comparison with our previous data ( $22.8 \%$ vs $35.5 \%$; $p<0.05$ ) showing a superimposable detection rate for cancer (18.7\% vs 22\%). Therefore, the significantly reduction of PSA levels in comparison with physiological PSA fluctuations (12) and the absence of reliable criteria to select patients at risk for $\mathrm{PCa}$ seems to suggest a prolonged trial of anti-inflammatory therapy before taking any decision in patients with negative prostate biopsy and persistently elevated PSA levels.

Our results deserve some considerations. Firstly, the absence of a placebo control group; for our purpose, we used as a surrogate our previously recorded cases. Secondly, it is unknown if PSA reduction is uniquely secondary to the anti-inflammatory activity or is in some way influenced by anti-proliferative effects of baicalina (25); thirdly, PCa incidence is available only in patients whose PSA levels remained unchanged after anti-inflammatory trial (the risk of missing PCa diagnosis in men with decreased PSA is actually unknown). Finally, a greater number of patients and a longer follow up are necessary to confirm our results.

In conclusion, in our preliminary data a prolonged oral anti-inflammatory therapy reduced PSA levels in selected patients (negative prostate biopsy and persistent elevated PSA values) decreasing the indication to perform repeat SPBx (about 30\% of the cases).

\section{REFERENCES}

1. Nixon RG, Lilly JD, Liedtke RJ, Batjer JD. Variation of free and total PSA levels: the effect on the percent free/total prostate antigen. Arch Pathol Lab Med. 1997; 121:385-91.

2. Yaman O, Gogus C, Tulunay $O$, et al. Increased PSA in subclinical prostatitis: the role of aggressiveness and extension of inflammation. Urol Int. 2003; 71:160-164.

3. Kandirali E, Boran C, Serin E. Association of extent and aggressiveness of inflammation with serum PSA levels and PSA density in asymptomatic patients. Urology. 2007; 70:743-747.

2. Hochreiter WW. The issue of prostate cancer evaluation in men with elevated PSA and chronic prostatitis. Andrologia. 2008; 40:130-133.

3. Stopiglia RM, Ferreira U, Silva MM, et al. PSA decrease and prostate cancer diagnosis: antibiotic versus placebo. Prospective randomized clinical trial. J Urol. 2010; 183:940-944.

4. Magri V, Trinchieri A, Montanari E, et al. Reduction of PSA values by combination pharmacological therapy in patients with chronic prostatitis: implications for prostate cancer detection. Arch It Urol Androl. 2007; 79:84-92.

5. Serretta V, Catanese A, Daricello G, et al. PSA reduction (after antibiotics) permits to avoid or postpone prostate biopsy in selected patients. Prostate Cancer Prostatic Diseases. 2008; 11:148-152.

6. Schaeffer AJ, Wu SC, Tennenberg AM, Kahn JB. Treatment of chronic bacterial prostatitis with levofloxacin and ciprofloxacin lowers serum PSA. J Urol. 2005; 174:161-164.

7. Terrone C, Poggio M, Bollito E, et al. Asymptomatic prostatitis: a frequent cause of raising PSA. Rec Prog Med. 2005; 96:365-369.

8. Erol H, Beder N, Caliskan T, et al. Can the effect of antibiotic therapy and anti-inflammatory therapy on serum PSA levels discriminate between benign and malign prostatic pathologies? Urol Int. 2006; 76:20-26.

9. Murad AS, Down L, Davey Smith G, et al. Associations of aspirin, nonsteroidal-antiflammatory drug and paracetamol use with PSAdetected prostate cancer: findings from a large, population-based, casecontrol study (the Protect study). Int J Cancer. 2011; 128:1442-48.

10. Pepe P, Aragona F. Incidence of insignificant prostate cancer using free/total PSA: results of a case-finding protocol on 14,453 patients. Prostate Cancer Prostatic Diseases. 2010; 13:316-319.

11. Pepe P, Aragona F. Saturation prostate needle biopsy and prostate cancer detection at initial and repeat evaluation. Urology. 2007; 70:1131-1135.

12. Boddy JL, Dev S, Pike DJ, Malone PR. Intra-individual variation of serum PSA levels in men with benign prostate biopsies. BJU Int. 2004; 93:735-38.

13. Nieder C, Norum J, Geinitz H. Impact of common medications on serum total PSA levels and risk group assignment in patients with prostate cancer. Anticancer Research. 2011; 31:1735-39.

14. Steven L. Chang, Lauren C. Harshman, and Joseph C. Presti Jr: Impact of common medications on serum total PSA levels: Analysis of the National Health and Nutrition Examination Survey J Clin Oncol. 2010; 28:3951-3957.

15. Nieder C, Norum J, Geinitz H. Impact of common medications 
on serum total PSA levels and risk group assignment in patients with prostate cancer. Anticancer Res. 2011; 31:1735-39.

16. Platz EA, Rohrmann S, Pearson JD, et al. Nonsteroidal antiinflammatory drugs and risk of prostate cancer in the Baltimore Longitudinal Study of Aging. Cancer Epidemiol Biomarkers Prev. 2005; 14:390-396.

17. Singer EA, Palapattu GS, van Wijngaarden E. PSA levels in relation to consumption of nonsteroidal anti-inflammatory drugs and acetaminophen: results from the 2001-2002 National Health and Nutrition Examination Survey. Cancer. 2008; 113:2053-7.

18. Mahmud S, Franco E, Aprikian A. Prostate cancer and use of nonsteroidal anti-inflammatory drugs: systematic review and metaanalysis. Br J Cancer. 2004; 90:93-99.

19. Harris RE, Beebe-Donk J, Doss H, Burr Doss D. Aspirin, ibuprofen, and other non-steroidal anti-inflammatory drugs in cancer prevention: a critical review of nonselective COX-2 blockade. Oncol Rep. 2005; 13:559-583.

20. Jacobs EJ, Thun MJ, Bain EB, et al. A large cohort study of long- term daily use of adult-strength aspirin and cancer incidence. J Natl Cancer Inst. 2007; 99:608-615.

21. Engelhardt PF, Rjedl CR. Effects of one-year treatment with isoflavone extract from red clover on prostate, liver function, sexual function, and quality of life in men with elevated PSA levels and negative prostate biopsy findings. Urology. 2008; 71:185-90.

22. Burnett BP, Jia Q, Zhao Y, Levy RM. A medical extract of Scutellaria baicalensis and Acacia catechu acts as dual inhibitor of ciclooxygenase to reduce inflammation. Journal of Medical Food. 2007; 442-451.

23. Lixuan Z, Jingcheng D, Wengin $Y$, et al. Baicalin attenuates inflammation by inhibiting NF-kappaB activation in cigarette smoke induced inflammatory models. Pulm Pharmacol Ther. 2010; 23:411-19.

24. Li C, Lin G, Zuo Z. Pharmacological effects and pharmacokinetics properties of radix Scutellariae and its bioactive flavones. Biopharm Drug Dispos. 2011; 32:427-45.

25. Marks LS, DiPaola RS, Nelson P, et al. herbal formulation for prostate cancer. Urology. 2002; 60:369-75.

\section{Correspondence}

Giuseppe Candiano, MD

Pietro Pepe, MD (Corresponding Author)

piepepe@hotmail.com

Francesco Pietropaolo, MD

Francesco Aragona, MD

Urology Unit - Cannizzaro Hospital, via Messina 829 - Catania, Italy 